\title{
Objective Quantum Gravity, Its Possible Relation to Gauge Theories and Strings
}

\author{
Lukasz Andrzej Glinka* \\ B.M. Birla Science Centre, Hyderabad, India \\ *Corresponding author: laglinka@gmail.com; lukaszglinka@wp.eu
}

Received May 24, 2014; Revised June 02, 2014; Accepted June 09, 2014

\begin{abstract}
In this paper the model of quantum gravity for the higher dimensional Lorentzian space-times, in the sense of the analogy with the Arnowitt-Deser-Misner decomposition well-known from General Relativity, is presented. The model is constructed through making use of the quantum geometrodynamics supplemented by the global onedimensionality conjecture, and considers the objective wave functionals. The framework of quantum field theory is applied in order to establish the phenomenological efficiency in accordance with high energy physics. The empirical deductions on the spatial dimensionality are presented as the relationship between the model and gauge theories, especially string theory.
\end{abstract}

Keywords: quantum gravity, quantum geometrodynamics, global one-dimensionality conjecture, objective functions, spatial dimension, string theory, Arnowitt-Deser-Misner decomposition

Cite This Article: Lukasz Andrzej Glinka, "Novel Solution of Wheeler-DeWitt Theory." Applied Mathematics and Physics, vol. 2, no. 3 (2014): 82-93. doi: 10.12691/amp-2-3-4.

\section{Introduction}

Recently quantum gravity has been denoted an increasing level of attention due to a number of theoreticians focused on seeking for the new physics. For this reason, the diverse approaches dedicated to this intriguing part of modern theoretical physics have already been emerged over the previ- ous forty years, particularly the most recent decade. Both the most pop- ularized and key models of quantum gravity are quantum geometrodynam- ics, Cf. the Refs. [1,2], perturbative quantum gravity, Cf. the Refs. [3,4,5], string theory, Cf. the Refs. [6,7,8], and loop quantum gravity, Cf. the Refs. $[9,10,11,12]$. However, in any of the well-known models of quantum gravity, one can eas- ily see both theoretical and technical difficulties in extraction of, potentially new, physical facts which could be able to be confronted with the available data of experimental high energy physics. This embarrassing state of affairs leads to manifestly nonphysical character of quantum gravity, and results in the shift of its considerations from theoretical physics towards evidently abstractive mathematics wherein the connections with physics are simply shadowed throughout the mishmash of diverse mathematical formalisms.

Actually, this problematic situation has already been become the dom- inating research trend in the numerous theoretical approaches, Cf. Ref. [13], which are overcrowded throughout mathematics instead of providing experimentally verifiable results. Strictly speaking, for the signifi- cant majority of quantum gravity researchers, extraction of physical facts is openly neglected or manifestly omitted in favour of developments in the purely methodological direction. In such a situation the most tremendous issue, probably having the epochal meaning for the study of quantum gravity, is to take the step forward towards finding the physics hidden in the math- ematical formalisms. Therefore, the major task of quantum gravity should be finding out the potentially empirically verifiable facts and reduction of the abstractive mathematical developments, because quantum gravity is re- garded as the branch of theoretical physics rather than pure mathematics. Nevertheless, after more than four decades since the moment of formula- tion the historically first model of quantum gravity, still there are unknown even the regions of energy wherein either quantum gravitation in itself or its detectable physical effects could be existent. The plausible and verifiable phenomenological approach could be based on quantum field theory, which lays the foundations of particle physics, because this would be leading to the transparent physical picture of this what really is quantum gravity, and, moreover, the results of this hypothetical approach could be automatically able to be empirically verified. However, the most popular models men- tioned above are very far from the successful implementation of the idea of confrontation between a mathematical formalism and physics.

Quantum geometrodynamics, the pioneering approach to quantization of Einstein's General Relativity investigated through the works of the Ameri- can scholars John Archibald Wheeler [14] and Bruce Selig- man DeWitt [15], is based on the method of quantization of the constrained Hamiltonian systems proposed by the a British Nobel Laureate in Physics Paul Adrien Maurice Dirac [16], and the Hamilto- nian formulation of General Relativity which for the first time was consid- ered by the 
American scholars Richard Lewis Arnowitt, Stanley Deser, and Charles William Misner [17]. The well-known problem of this model of quantum gravity is the functional-differential evolution known as the WheelerDeWitt equation. For the case of a $D$-dimensional space embedded into a $D+1$-dimensional enveloping spacetime, the embarrassing functional differentiation is taken with respect to $D \times D$-dimensional symmetric matrix following from the ADM decomposition of a space-time metric. Therefore, the level of computational complexity in quantum geometrody- namics is truly tremendous, because one must solve $D(D+1) / 2$ functional differential equations which, from the formal point of view of theory of dif- ferential equations, have no a strictly defined sense. In this manner, both the physical and mathematical nature of the Wheeler-DeWitt wave functionals remain a great riddle of the mathematical mishmash. In fact, the crucial com- plications arise due to the configuration space of General Relativity, known as the Wheeler superspace and containing the points being all possible ge- ometries of an embedded space, which defines the abstractive structure of quantum geometrodynamics. The only known class of solutions are the wave functionals constructed in the strict analogy to the semi-classical Wentzel- Kramers-Brillouin wave functions, determined throughout the Feynman path integral method, which are well-known as the HartleHawking wave functions [18]. Nevertheless, the WKB states are computable for the simplest situations merely related to cosmological models, Cf. the Refs. [19] and [20], and their merely ide- ological applicability recently has been developed significantly in the new string theory-like directions, Cf. the Ref. [21].

In this paper, the quantum gravity model of higher dimensional Lorentzian space-times formulated within the framework of quantum geometrodynamics and in terms of quantum field theory, strictly based on the previous works of the author [22], is presented.

\section{Quantum Geometrodynamics}

Let us consider a space-time modelled through a D + 1dimensional pseudo-Riemannian manifold $(M, g)$ equipped with D +1 -volume form $g=\operatorname{det} g_{\mu \nu}$ defined by a metric $g_{\mu \nu}$ of the Lorentzian signature $(1, D)$, the Christoffel symbols $\Gamma_{\mu v}^{\rho}$, the Riemann-Christoffel curvature tensor $R_{\mu \alpha v}^{\lambda}$, the Ricci curvature tensor $R_{\mu v}$, and the Ricci scalar curvature ${ }^{(D+1)} R$

$$
\begin{gathered}
\Gamma_{\mu \nu}^{\rho}=\frac{1}{2} g^{\rho \sigma}\left(g_{\mu \sigma, v}+g_{\sigma v, \mu}-g_{\mu v, \sigma}\right), \\
R_{\mu \alpha v}^{\lambda}=\Gamma_{\mu \nu, \alpha}^{\lambda}-\Gamma_{\mu \alpha, v}^{\lambda}+\Gamma_{\sigma \alpha}^{\lambda} \Gamma_{\mu \nu}^{\sigma}-\Gamma_{\sigma \nu}^{\lambda} \Gamma_{\mu \alpha}^{\sigma}, \\
R_{\mu v}=R_{\mu \lambda v}^{\lambda}=\Gamma_{\mu v, \lambda}^{\lambda}-\Gamma_{\mu \lambda, v}^{\lambda}+\Gamma_{\sigma \lambda}^{\lambda} \Gamma_{\mu \nu}^{\sigma}-\Gamma_{\sigma \nu}^{\lambda} \Gamma_{\mu \lambda}^{\sigma}, \\
{ }^{(D+1)} R=g^{\mu v} R_{\mu \nu} .
\end{gathered}
$$

General Relativity, Cf. the Refs. [23,24,25,26,27], is defined by the Einstein field equations

$$
G_{\mu v}=\Lambda g_{\mu v}=\kappa T_{\mu v},
$$

where

$$
G_{\mu v}=R_{\mu v}-\frac{1}{2} g_{\mu \nu}{ }^{(D+1)} R,
$$

is the Einstein tensor, $\Lambda$ is the cosmological constant, non-stationary Matter fields are reflected through a nonvanishing stress-energy tensor $T_{\mu \nu}$, and $\kappa=8 \pi G / c^{4} \approx 2.08 \cdot 10^{-43} \mathrm{~N}^{-1}$ is the Einstein constant, with $G$ being the Newton gravitational constant and $c$ being the speed of light in vacuum.

The Hamiltonian formulation of General Relativity is easily generalizable to a D+1-dimensional space-time of the Lorentzian signature $(1, D)$. This is necessary to foliate a space-time manifold $M$ with slices, that is a family of space-like hypersurfaces, and this is perforable when $M$ is a globally hyperbolic manifold, but all Lorentzian space-times are of this type. If there are given a scalar field $t\left(x^{\mu}\right)$ on $M$, such that the foliation $t=$ constans defines a family of non-intersecting space-like hypersurfaces $\Sigma(t)$, the coordinate system $y^{i}$ on all $\Sigma$, and a fixed hypersurface $\Sigma$ defined through the system of parametric equations $x^{\mu}=x^{\mu}\left(y^{i}\right)$, where the index $i=1, \ldots, D$ labels coordinates intrinsic to $\Sigma$, or, equivalently, a restriction $f\left(x^{\mu}\right)=0$, then $\partial_{\mu} f\left(x^{\mu}\right)$ is a normal to $\sum$ which, if is not null, defines the unit normal vector field to $\Sigma$ as a future-directed time-like vector field $n_{\mu} \sim \partial_{\mu} t$ satisfying $n^{\mu} n_{\mu}=-1$

$$
n_{\mu}=-\frac{\partial_{\mu} f}{\sqrt{\left|\partial_{\mu} f \partial^{\mu} f\right|}}, \quad n^{\mu} \partial_{\mu} f>0 .
$$

Let $\gamma$ be a congruence of curves intersecting $\sum(t)$ 's which in general are not geodesics nor orthogonal to $\Sigma(t)$. Let $\mathrm{t}$ be a parameter on $\gamma$, and $t^{\mu}$ a tangent vector to $\gamma$. Then,

$$
t^{\mu} \partial_{\mu} t=1 \text {. }
$$

An arbitrary fixed curve $\gamma_{F}$ is a mapping between points on all hypersurfaces $\sum(t)$

$$
\begin{aligned}
& \gamma_{F}: P \in \sum(t) \mapsto P^{\prime} \in \sum\left(t^{\prime}\right) \mapsto P^{\prime \prime} \in \sum\left(t^{\prime \prime}\right) \\
& \mapsto \ldots P^{(n)} \in \sum\left(t^{(n)}\right), \quad n \in \mathbb{Z}_{+}
\end{aligned}
$$

and fixation of coordinates on any two $\Sigma_{s}$ establishes constant coordinates $y^{i}$ for each $n$. This determines the coordinate system $\left(t, y^{i}\right)$ in $M$. If there is another coordinate system $x^{\mu}: x^{\mu}\left(t, y^{i}\right)$, then $t^{\mu}$ and the tangent vectors $e_{i}^{\mu}$ on any $\Sigma(t)$ in $\left(t, y^{i}\right)$ are 


$$
t^{\mu}=\left(\partial_{\mu} x^{\mu}\right)_{y^{i}}=\delta_{t}^{\mu}, e_{i}^{\mu}=\left(\partial_{y^{i}} x^{\mu}\right)_{t}=\delta_{i}^{\mu}
$$

In any coordinates the Lie derivative of the tangent vectors vanishes, that is $\mathcal{L}_{t} e_{i}^{\mu}=0$ holds. Let us consider the unit normal vector field $n_{\mu}$ to $\sum(t)$

$$
n_{\mu}=-N \partial_{\mu} t, \quad n_{\mu} e_{i}^{\mu}=0,
$$

where $N$, called the lapse scalar, normalizes $n_{\mu}$. In general, $t^{\mu}, t^{\mu} \nVdash n^{\mu}$ and, therefore, $t^{\mu}$ can be decomposed in the basis $\left(n_{\mu}, e_{i}^{\mu}\right)$

$$
t^{\mu}=N n^{\mu}+N^{i} e_{i}^{\mu},
$$

where $N^{i}$ is called the shift vector. The transformation $x^{\mu}=x^{\mu}\left(t, y^{i}\right)$ allows to write in $\left(t, y^{i}\right)$

$$
\begin{aligned}
& d x^{\mu}=t^{\mu} d t+e_{i}^{\mu} d y^{i}=\left(N n^{\mu}+N^{i} e_{i}^{\mu}\right) d t+e_{i}^{\mu} d y^{i} \\
& =(N d t) n^{\mu}+\left(d y^{i}+N^{i} d t\right) e_{i}^{\mu},
\end{aligned}
$$

$$
\begin{aligned}
& \text { and } \begin{array}{c}
\text { hence the space-time } \\
d s^{2}=g_{\mu v} d x^{\mu} d x^{v}=d x^{\mu} d x_{\mu} \text { is }
\end{array} \\
& \qquad \begin{aligned}
d s^{2}= & -\left(N^{2}-N_{i} N^{i}\right) d t^{2}+N_{i} d x^{i} d t \\
& +N_{j} d x^{j} d t+h_{i j} d x^{i} d x^{j},
\end{aligned}
\end{aligned}
$$

where $h_{i j}$ is an induced metric on $\sum(t)$

$$
h_{i j}=g_{\mu v} e_{i}^{\mu} e_{j}^{v} .
$$

The decomposition (14), for the special case $D=3$, was investigated in [17]. A space-time metric $g_{\mu v}$ satisfying the Einstein field equations (5) is therefore

$$
\begin{aligned}
& g_{\mu v}=\left[\begin{array}{cc}
-N^{2}+N_{i} N^{i} & N_{j} \\
N_{i} & h_{i j}
\end{array}\right], \\
& g^{\mu v}=\frac{1}{N^{2}}\left[\begin{array}{cc}
-1 & N^{j} \\
N^{i} & N^{2} h^{i j}-N^{i} N^{j}
\end{array}\right],
\end{aligned}
$$

where $N^{j}=h^{i j} N_{i}$ is the contravariant shift vector, and the spatial metric satisfies the orthogonality condition

$$
h_{i k} h^{k j}=\delta_{i}^{j} \text {. }
$$

Completeness relations are

$$
g_{\mu v}=-n_{\mu} n_{v}+h_{i j} e_{\mu}^{i} e_{v}^{j}, g^{\mu v}=-n^{\mu} n^{v}+h^{i j} e_{i}^{\mu} e_{j}^{v} .
$$

It can be verified straightforwardly that the transformation between the D+1-volume form and the Dvolume form is

$$
\sqrt{g}=N \sqrt{h}
$$

The second fundamental form of a slice, called the extrinsic curvature tensor or induced curvature, is

$$
K_{i j}=n_{\mu ; \nu} e_{i}^{\mu} e_{j}^{\nu}=-\nabla_{(i} n_{j)}-n_{(i} n^{k} n_{j) \mid k},
$$

and its trace, called the intrinsic curvature, has the form

$$
K=K_{i}^{i}=h^{i j} K_{i j}=n_{; \mu}^{\mu} .
$$

$\sum$ is convex when congruence is diverging, that is $K>0$, and concave when the congruence is converging, that is $K<0$. The tangent vectors to $\Sigma(t)$ satisfy the Gauss-Weingarten equations

$$
e_{i ; \beta}^{\alpha} e_{j}^{\beta}=\Gamma_{i j}^{k} e_{k}^{\alpha}+K_{i j} n^{\mu},
$$

and the Gauss-Codazzi equations can be derived straightforwardly

$$
\begin{gathered}
R_{\mu v \kappa \lambda} e_{i}^{\mu} e_{j}^{v} e_{k}^{\kappa} e_{l}^{\lambda}=R_{i j k l}-K_{i l} K_{j k}+K_{i k} K_{j l}, \\
R_{\mu v \kappa \lambda} n^{\mu} e_{i}^{v} e_{j}^{\kappa} e_{k}^{\lambda}=K_{i j \mid k}-K_{i k \mid j} .
\end{gathered}
$$

Applying the decomposition of the Ricci curvature tensor and Ricci scalar curvature

$$
R_{\mu \nu}=-R_{\kappa \mu \lambda \nu} n^{\kappa} n^{\lambda}+h^{i j} R_{\kappa \mu \lambda \nu} e_{i}^{\kappa} e_{j}^{\lambda},
$$

$$
\begin{aligned}
{ }^{(D)} R= & -2 h^{k l} R_{\kappa \mu \lambda \nu} n^{\kappa} n^{\lambda} e_{k}^{\mu} e_{l}^{v} \\
& +h^{k l} h^{i j} R_{\kappa \mu \lambda \nu} e_{i}^{\kappa} e_{j}^{\lambda} e_{k}^{\mu} e_{l}^{v},
\end{aligned}
$$

one receives the projections of the Einstein tensor

$$
\begin{gathered}
2 G_{\mu v} n^{\mu} n^{v}={ }^{(D)} R+K^{i j} K_{i j}+K^{2}, \\
G_{\mu v} e_{j}^{\mu} n^{v}=K_{j \mid i}^{i}-K_{, j} .
\end{gathered}
$$

Another identity, called the Ricci equation

$$
\mathcal{L}_{n} K_{i j}=n^{\mu} n^{v} e_{i}^{\kappa} e_{j}^{\lambda} R_{\mu v \kappa \lambda}-\frac{1}{N} N_{\mid i j}-K_{i k} K_{j}^{k},
$$

can be also easy derived. The stroke on the left of an index means the intrinsic covariant differentiation with respect to a coordinate labeled by this index. Two indices before the stroke means taking two times the intrinsic covariant derivative with respect to each of the indices. For instance for a vector $V_{i}$ and a tensor $T_{i j}$ one has

$$
\begin{gathered}
V_{i \mid j}=\nabla_{j} V_{i}=\partial_{j} V_{i}-\Gamma_{j i}^{k} V_{k}, \\
T_{i j \mid k}=\nabla_{k} T_{i j}=\partial_{k} T_{i j}-T_{l j} \Gamma_{i k}^{l}-T_{i l} \Gamma_{j k}^{l},
\end{gathered}
$$

where $\Gamma_{i j}^{k}$ are the spatial Christoffel symbols

$$
\Gamma_{i j}^{k}=\frac{1}{2} h^{k l}\left(h_{i l, j}+h_{l j, i}-h_{i j, l}\right) .
$$

Using the completeness relations (18) for the inverted metric and the fact

$$
R_{\kappa \mu \lambda v} n^{\kappa} n^{\mu} n^{\lambda} n^{v}=0,
$$

one sees that the first term in (26) reduces to $-2 R_{\mu v} n^{\mu} n^{v}$. Application of 


$$
\begin{gathered}
R_{\mu v} n^{\mu} n^{v}=2\left(n_{;[v}^{\mu} n^{v}\right)_{; \mu]}+2 n_{;[\mu}^{\mu} n_{; v]}^{v}, \\
n_{; \nu}^{\mu} n_{; \mu}^{v}=K^{i j} K_{i j},
\end{gathered}
$$

to the first term in (26), and the equations (23) to the second one

$$
\begin{aligned}
& h^{k l} h^{i j} R_{\kappa \mu \lambda \nu} e_{i}^{\kappa} e_{j}^{\lambda} e_{k}^{\mu} e_{l}^{v} \\
= & h^{k l} h^{i j}\left(R_{i j k l}-K_{i l} K_{j k}+K_{i k} K_{j l}\right) \\
= & { }^{(D)} R+K^{2}-K^{i j} K_{i j},
\end{aligned}
$$

leads to the D-dimensional evaluation of the $\mathrm{D}+1$ dimensional Ricci scalar curvature

$$
{ }^{\left({ }^{D+1}\right)} R={ }^{(D)} R+K^{2}-K^{i j} K_{i j}-2\left(n_{; v}^{\mu} n^{v}-n^{\mu} n_{; v}^{v}\right)_{; \mu} .
$$

The pair of dynamical variables - induced metric $h_{i j}$ and extrinsic curvature $K_{i j}$ - describes geometry of a submanifold $\partial M$ by the D+1-decomposed Einstein field equations, and the local geometry of a single space-like hypersurface $\partial M$. Evolution of the global space-time geometry can be formulated through the one-parameter family $\left(h_{i j}(t), K_{i j}(t)\right)$ describing the dynamics of local space geometry of the constant time hypersurfaces $\partial M_{t}$. For consistency, the relation between the time evolution operator $\partial_{t}$ and the vector field n normal to $\partial M_{t}$ must be specified

$$
\partial_{t}=N n+N^{i} \partial_{i}
$$

Let an enveloping space-time manifold $M$ be compact and possesses a space-like boundary $(\partial M, h)$ equipped with a D-volume form $h=\operatorname{det} h_{i j}$ defined by an induced metric $h_{i j}$, and the second fundamental form $K_{i j}$. Let topology of $\mathrm{M}$ be $\Sigma \times \mathbb{R}$, where $\Sigma$ is an unrestricted topology of $\partial M$. Then, the Einstein field equations (5) are the Euler-Lagrange equations of motion following from the Hilbert-Palatini action principle with respect to the fundamental field $g_{\mu v}$ supplemented by the suitable boundary condition

$$
\frac{\delta S[g]}{\delta g_{\mu v}}=0, \delta g_{\mu v \mid \partial M}=0,
$$

and applied to the Einstein-Hilbert action complemented through the York-Gibbons-Hawking boundary action, that is the action of a D + 1-geometry with fixed an induced D-geometry of a boundary

$$
\begin{aligned}
S[g]= & \frac{1}{2 \kappa c} \int_{M} d^{D+1} x \sqrt{-g}\left(-{ }^{(D+1)} R+2 \Lambda\right) \\
& -\frac{1}{\kappa c} \int_{\partial M} d^{D} x \sqrt{h} K+S_{\phi}[g],
\end{aligned}
$$

where $S_{\phi}[g]$ is the action of Matter fields

$$
S_{\phi}[g]=\frac{1}{c} \int_{M} d^{D+1} x \sqrt{-g} L_{\phi}
$$

The Einstein field equations (5) result from vanishing of the variation $\delta S=\delta S_{G}+\delta S_{\phi}=0$ on $\partial M$, where $S_{G}=S_{E H}+S_{Y G H}$ and

$$
\begin{gathered}
\delta S_{G}=\frac{1}{2 \kappa c} \int_{M} d^{D+1} x \sqrt{-g}\left(G_{\mu v}+\Lambda g_{\mu v}\right) \delta g^{\mu \nu}, \\
\delta S_{Y G H}=\frac{1}{2 \kappa c} \int_{\partial M} d^{D} y \sqrt{|h|} h^{\mu v} n^{\rho} \delta g_{\mu v, \rho}, \\
\delta S=-\frac{1}{2 c} \int_{M} d^{D+1} x \sqrt{-g} T_{\mu \nu} \delta g^{\mu \nu} .
\end{gathered}
$$

Moreover, the variational principle establishes the relationship

$$
T_{\mu \nu}=-\frac{2}{\sqrt{g}} \frac{\delta}{\delta g^{\mu \nu}}\left(\sqrt{-g} L_{\phi}\right) .
$$

In general, stationarity of Matter fields, that is $T_{\mu v} \equiv 0$, results in existence of a global time-like Killing vector field $\mathcal{K}_{\mu}$ for a metric tensor $g_{\mu \nu}$. Recall, Cf. the Ref. [23], that such a field obeys the equation

$$
\mathcal{L}_{K} g_{\mu \nu}=\lim _{\epsilon \rightarrow 0} \frac{g_{\mu \nu}(\tilde{x})-\tilde{g}_{\mu \nu}(\tilde{x})}{\epsilon}=0,
$$

where $\tilde{g}_{\mu v}(\tilde{x})$ is a metric $g_{\mu v}(x)$ transformed under the isometric mapping

$$
\tilde{x}^{\mu}=x^{\mu}+\epsilon \mathcal{K}^{\mu}
$$

which is equivalent to the Killing equation

$$
\nabla_{(\mu} \mathcal{K}_{v)}(x)=0
$$

In other words, the Killing vector fields are the infinitesimal generators of isometries. One can specify a coordinate system such that $\mathcal{K}_{\mu}=\left[\partial_{t}, 0,0,0\right]$ and the foliation $t=$ constans is space-like. In such a situation, $g_{\mu v}$ depends at most on a spatial coordinates xi, and time $\mathrm{t}$ is a global coordinate [28]. Let us specify this coordinate in such a way that an induced space is a $t=$ constans hypersurface. Then, $\partial M$ satisfies the conditions of the Nash embedding theorem, Cf. the Refs. [29]. If $\Lambda=0$, then a global time-like Killing vector field on $M$ exists. When $\Lambda>0$, then $\mathcal{K}_{\mu}$ does not exist, and space-like boundary $\partial M$ only foliates an exterior to the horizons on geodesic lines. Then $\mathrm{D}+1$ decomposition (16) is a gauge of the fundamental field $g_{\mu v}$.

The action (40) evaluated for a D + 1 metric (16) takes the Hamiltonian form

$$
S[g]=\int d t L .
$$

The Lagrangian related to the York-Gibbons-Hawking boundary action is a total derivative, and, therefore, not essential in the analysis. Contributions due to Matter fields and cosmological constant are simple to analysis. The most complicated is the geometric part of the EinsteinHilbert Lagrangian 


$$
\begin{aligned}
& \sqrt{g}\left(-{ }^{(D+1)} R+2 \Lambda\right) \\
= & N \sqrt{h}\left(-K^{i j} K_{i j}+K^{2}-{ }^{(D)} R+2 \Lambda\right) \\
+ & 2 \partial_{0}(\sqrt{h} K)-2 \partial_{i}\left(\sqrt{h}\left(K N^{i}-h^{i j} N_{\mid j}\right)\right),
\end{aligned}
$$

and because the last two terms are total derivatives they can be dropped when performing a canonical formulation. In result, one obtains the Lagrangian

$$
L=\frac{1}{2 \kappa} \int_{\partial M} d^{D} x N \sqrt{h}\left(\begin{array}{l}
K^{2}-K^{i j} K_{i j} \\
-(D) R+2 \Lambda+2 \kappa \rho
\end{array}\right) .
$$

The D + 1-decomposed Einstein field equations can be derived easily, and describe the evolutionof an induced metric $h_{i j}$ and the intrinsic curvature $K_{i j}$

$$
\begin{gathered}
\partial_{t} h_{i j}=N_{i \mid j}+N_{j \mid i}-2 N K_{i j}, \\
\partial_{t} K_{i j}=-N_{\mid j j}+N\left(R_{i j}+K K_{i j}-2 K_{i k} K_{j}^{k}\right) \\
+N^{k} K_{i j \mid k}+K_{i k} N_{\mid j}^{k}+K_{j k} N_{\mid i}^{k} \\
-\kappa N\left[S_{i j}-\frac{S-\varrho}{D-2} h_{i j}\right] .
\end{gathered}
$$

Here $\varrho$, called the energy density, is a double projection of the stress-energy tensor onto the normal vector field

$$
\varrho=T(n, n)=T_{\mu v} n^{\mu} n^{v},
$$

and $n^{\mu}$ is the normal vector field following from the D + 1 splitting

$$
n^{\mu}=\left[\frac{1}{N},-\frac{N^{i}}{N}\right], n_{\mu}=[-N, 0 \ldots, 0]^{T} .
$$

Spatial stress tensor $S_{i j}$ is s double projection of the stress-energy tensor onto the spatial metric, and spatial stress density $S$ is its trace

$$
S_{i j}=T(h, h)=T_{\mu \nu} h_{i}^{\mu} h_{j}^{v}, S=h^{i j} S_{i j},
$$

where $h_{v}^{\mu}=\delta_{v}^{\mu}+n^{\mu} n_{v}$. The Lagrangian (52) leads to the Euler-Lagrange equations of motion

$$
\begin{aligned}
& 2 \kappa h^{-1}\left(h_{i k} h_{j l}-\frac{1}{2} h_{i j} h_{k l}\right) \frac{\delta S}{\delta h_{i j}} \frac{\delta S}{\delta h_{k l}} \\
& -\frac{1}{2 \kappa}\left({ }^{(D)} R+2 \Lambda+2 \kappa \varrho\right)=0,
\end{aligned}
$$

and

$$
\pi_{\mid j}^{i j}+J^{i}=0
$$

where the momentum $\pi^{i j}$ conjugated to the induced metric is

$$
\pi^{i j}=\frac{\delta S[g]}{\delta h_{i j}}=\frac{\delta L}{\delta\left(\partial_{t} h_{i j}\right)}=-\frac{1}{2 \kappa} \sqrt{h}\left(K^{i j}-h^{i j} K\right),
$$

and the momentum density $J^{i}$ is the stress-energy tensor projected onto the normal vector field and the spatial metric

$$
J^{i}=T(n, h)=T_{\mu v} n^{\mu} h^{v i} .
$$

The equation (58) is the Hamilton-Jacobi equation of the D+1-dimensional General Relativity and defines the classical geometrodynamics.

Let us analyze the Lagrangian (52). The canonical momenta of the theory

$$
\begin{gathered}
\pi_{\phi}=\frac{\delta L}{\delta\left(\partial_{t} \phi\right)}, \\
\pi=\frac{\delta L}{\delta\left(\partial_{t} N\right)}=0, \\
\pi^{i}=\frac{\delta L}{\delta\left(\partial_{t} N_{i}\right)}=0,
\end{gathered}
$$

allow to make the Legendre transformation of the Lagrangian

$L=\int_{\partial M} d^{D} x\left[\frac{1}{2 \kappa}\left(\begin{array}{c}\pi_{\phi} \partial_{t} \phi+\pi \partial_{t} N \\ +\pi^{i} \partial_{t} N_{i}+\pi^{i j} \partial_{t} h_{i j}\end{array}\right)-N H-N_{i} H^{i}\right]$,

where the quantities $H$ and $H^{i}$ are defined as

$$
\begin{aligned}
H & =\frac{\sqrt{h}}{2 \kappa}\left(K^{2}-K_{i j} K^{i j}-{ }^{(D)} R+2 \Lambda+2 \kappa \varrho\right), \\
H^{i} & =-2 \pi^{i j} \mid j-2 J^{i} \\
& =-2 \partial_{j} \pi^{i j}-h^{i l}\left(2 h_{j l, k}-h_{j k, l}\right) \pi^{j k}-2 J^{i},
\end{aligned}
$$

Application of the time-preservation to the primary constraints

$$
\pi \approx 0, \pi^{i} \approx 0
$$

lead to the secondary constraints

$$
\begin{aligned}
H & \approx 0, \\
H^{i} & \approx 0,
\end{aligned}
$$

called the Hamiltonian (scalar) constraint which yields the dynamics, and the diffeomorphism (vector) constraint which merely reflects the spatial diffeoinvariance. The quantities $H^{i}$ generate the spatial diffeomorphisms $\tilde{x}^{i}=x^{i}+\xi^{i}$

$$
\begin{array}{rl} 
& i\left[h_{i j}, \int_{\partial M} H_{a} \xi^{a} d^{D} x\right] \\
= & -h_{i j, k} \xi^{k}-h_{k j} \xi^{k}, i-h_{i k} \xi^{k},{ }_{, j}, \\
i & i\left[\pi_{i j}, \int_{\partial M} H_{a} \xi^{a} d^{D} x\right] \\
= & -\left(\pi^{i j} \xi^{k}\right)_{, k}+\pi^{k j} \xi_{, k}^{i}+\pi^{i k} \xi_{, k}^{j},
\end{array}
$$

where $H_{i}=h_{i j} H^{j}$. Application of the structure constants of the diffeomorphism group 


$$
c_{i j}^{a}=\delta_{i}^{a} \delta_{j}^{b} \delta_{, b}^{(D)}(x, z) \delta^{(D)}(y, z)-(x \rightarrow y),
$$

to the relations (71) and (72) leads to the first-class constraints algebra

$$
\begin{gathered}
i\left[H_{i}(x), H_{j}(y)\right]=\int_{\partial M} H_{a} c_{i j}^{a} d^{D} z, \\
i\left[H(x), H_{i}(y)\right]=H \delta_{, i}^{D}(x, y),
\end{gathered}
$$

while involving of the elementary relation

$$
\begin{aligned}
\delta\left(\sqrt{h}^{(D)} R\right)= & \sqrt{h} h^{i j} h^{k l}\left(\delta h_{i k, j l}-\delta h_{i j, k l}\right) \\
& -\sqrt{h}\left[R^{i j}-\frac{1}{2} h^{i j(D)} R\right] \delta h_{i j},
\end{aligned}
$$

allows to establish the third bracket

$$
\begin{aligned}
& i\left[\int_{\partial M} H \xi_{1} d^{D} x, \int_{\partial M} H \xi_{2} d^{D} x\right] \\
= & \int_{\partial M} H^{a}\left(\xi_{1, a} \xi_{2}-\xi_{1} \xi_{2, a}\right) d^{D} x .
\end{aligned}
$$

Primary quantization method [16]

$$
\begin{gathered}
i\left[\pi^{i j}(x), h_{k l}(y)\right]=\frac{1}{2}\left(\delta_{k}^{i} \delta_{l}^{j}+\delta_{l}^{i} \delta_{k}^{j}\right) \delta^{(D)}(x, y), \\
i\left[\pi^{i}(x), N_{j}(y)\right]=\delta_{j}^{i} \delta^{(D)}(x, y), \\
i[\pi(x), N(y)]=\delta^{(D)}(x, y) .
\end{gathered}
$$

in the Wheeler metric representation, gives

$$
\pi=-i \frac{\delta}{\delta N}, \pi^{i}=-i \frac{\delta}{\delta N_{i}}, \pi^{i j}=i \frac{\delta}{\delta h_{i j}},
$$

which applied in the constraint (66) yield the WheelerDeWitt equation

$$
\left\{\begin{array}{l}
2 \kappa G_{i j k l} \frac{\delta^{2}}{\delta h_{i j} \delta h_{k l}} \\
+\frac{1}{2 \kappa} \sqrt{h}\left({ }^{(D)} R-2 \Lambda-2 \kappa \varrho\right)
\end{array}\right\} \Psi\left[h_{i j}, \phi\right]=0,
$$

where $G_{i j k l}$ is the DeWitt supermetric on the Wheeler superspace

$$
G_{i j k l}=\frac{1}{2 \sqrt{h}}\left(h_{i k} h_{j l}+h_{i l} h_{j k}-h_{i j} h_{k l}\right),
$$

Cf. the Ref. [30]. Other first-class constraints satisfy

$$
\begin{gathered}
{\left[\pi(x), \pi^{i}(y)\right]=0,\left[\pi(x), H^{i}(y)\right]=0} \\
{\left[\pi^{i}(x), H^{j}(y)\right]=0,\left[\pi^{i}(x), H(y)\right]=0,}
\end{gathered}
$$

and in the canonical quantization are the supplementary conditions on $\Psi\left[h_{i j}, \phi\right]$. Diffeoinvariance is reflected by the primary constraints and the diffeomorphism constraint which give

$$
\begin{aligned}
& -i \frac{\delta \Psi\left[h_{i j}, \phi\right]}{\delta N}=0,-i \frac{\delta \Psi\left[h_{i j}, \phi\right]}{\delta N_{i}}=0, \\
& -i\left(\frac{\delta \Psi\left[h_{i j}, \phi\right]}{\delta h_{i j}}\right)_{\mid j}=J^{i} \Psi\left[h_{i j}, \phi\right] .
\end{aligned}
$$

\section{Global Optimization in Higher Dimensions}

Geometry of an enveloping space-time can be evaluated by an embedding geometry, whose characteristics are functionals of an induced metric $h_{i j}$. Spatial stress density and cosmological constant are also functionals of $h_{i j}$ and, therefore, energy density of Matter fields $\varrho$ must be a functional of $h_{i j}$. This situation implies the state of affairs wherein Matter fields are functionals of $h_{i j}$. In this manner, the DeWitt supposition [15]

$$
\Psi\left[h_{i j}, \phi\right] \equiv \Psi\left[\mathfrak{G}^{D}\right],
$$

holds. The problem is solving the Wheeler-DeWitt equation, and it should be emphasized that the only semiclassical solutions [18] are known. Even $\Psi\left[\mathfrak{G}^{D}\right]=\Psi\left[h_{i j}\right]$ does not simplify the situation, there is still a functional dependence on a $D \times D$ matrix $h_{i j}$, and it is not clear how to treat $\Psi\left[h_{i j}\right]$. In general, the Wheeler-DeWitt equation, as the result of the primary canonical quantization, defines a quantum mechanics and, for this reason, $\Psi\left[h_{i j}\right]$ should be a scalar functional in $h_{i j}$. The Wheeler-DeWitt operator is $\operatorname{Diff}(\partial M)$-invariant and, consequently, the full invariance is assured if and only if $\Psi\left[h_{i j}\right]$ is a diffeoinvariant, that is a function of $\operatorname{Diff}(\partial M)$ invariants which must be constructed from $h_{i j}$ only.

\subsection{Global One-Dimensionality Conjecture}

Through a generalization of DeWitt's construction [15], $\Psi\left[h_{i j}\right]$ is a functional of the $D \times D$ symmetric matrix $h_{i j}$. The analogy to quantum mechanics suggests that $\Psi\left[h_{i j}\right]$, as a wave function, is a classical scalar field, that is a single functional. Consequently, one shall to perform the global optimization and, for this reason, $\Psi\left[h_{i j}\right]$ should be dependent on the matrix invariants $c_{n}$ of $h_{i j}=$ h. In the light of the Cayley-Hamilton theorem, $c_{n}$ are the coefficients of the characteristic polynomial of $h_{i j}$

$$
\mathrm{h}^{D}-c_{1} \mathrm{~h}^{D-1}-c_{2} \mathrm{~h}^{D-2}-\cdots+c_{D} \mathrm{I}_{D}=0,
$$

where $\mathrm{I}_{D}$ is the $D \times D$-dimensional unit matrix. For the case $k=1, \ldots, D-1, \quad c_{k}$ can be established recursively by the Newton identities

$$
k c_{k}=p_{k}-\sum_{i=1}^{k-1} c_{i} p_{k-i},
$$

where $p_{k}=T r h^{k}$ and, consequently,

$$
\begin{gathered}
c_{1}=p_{1}, \\
c_{2}=\frac{1}{2} p_{2}-\frac{1}{2 !} p_{1}^{2},
\end{gathered}
$$




$$
\begin{gathered}
c_{3}=\frac{1}{3} p_{3}-\frac{1}{2} p_{1} p_{2}+\frac{1}{3 !} p_{1}^{3}, \\
c_{4}=\frac{1}{4} p_{4}-\frac{1}{3} p_{1} p_{3}+\frac{1}{4} p_{1}^{2} p_{2}-\frac{1}{2 \cdot 4} p_{2}^{2}-\frac{1}{4 !} p_{1}^{4}, \\
c_{5}=\frac{1}{5} p_{5}-\frac{1}{4} p_{1} p_{4}-\frac{1}{6} p_{2} p_{3}-\frac{1}{12} p_{1}^{3} p_{2} \\
+\frac{1}{6} p_{1}^{2} p_{3}+\frac{1}{8} p_{1} p_{2}^{2}+\frac{1}{5 !} p_{1}^{5},
\end{gathered}
$$

and so on. For $k=D$ the coefficient has the form

$$
c_{D}=(-1)^{D} \operatorname{det} \mathrm{h} .
$$

A scalar valued matrix function $\Psi\left(h_{i j}\right)$ that depends merely on $c_{k}$

$$
\Psi\left(h_{i j}\right)=\Psi\left(c_{1}, c_{2}, \ldots, c_{D}\right),
$$

remains unchanged under rotations of a coordinate system and is known as an objective function and, therefore, the wave functions (96) define objective higher dimensional quantum gravity. In this manner, we shall consider

$$
\left\{\begin{array}{l}
2 \kappa G_{i j k l} \frac{\delta^{2}}{\delta h_{i j} \delta h_{k l}} \\
+\frac{h^{1 / 2}}{2 \kappa}\left({ }^{(D)} R-2 \Lambda-2 \kappa \varrho[h]\right)
\end{array}\right\} \Psi\left(c_{1}, c_{2}, \ldots, c_{D}\right)=0,(97)
$$

that is quantum geometrodynamics on the objective strata of the superspace.

\subsection{Objective Quantum Geometrodynamics}

The whole problem is contained in the operator

$$
G_{i j k l} \frac{\delta^{2}}{\delta h_{i j} \delta h_{k l}} \Psi\left(c_{1}, c_{2}, \ldots, c_{D}\right) .
$$

Let us consider the obvious identity

$$
\frac{\delta}{\delta h_{i j}} \Psi\left(c_{1}, c_{2}, \ldots, c_{D}\right)=\sum_{n=1}^{D} \frac{\delta c_{n}}{\delta h_{i j}} \frac{\delta}{\delta c_{n}} \Psi\left(c_{1}, c_{2}, \ldots, c_{D}\right)(99)
$$

which together with the chain differentiation rule

$$
\frac{\delta}{\delta c_{n}} \Psi\left(c_{1}, c_{2}, \ldots, c_{D}\right)=\frac{\delta h}{\delta c_{n}} \frac{\delta}{\delta h} \Psi(h),
$$

gives

$$
\frac{\delta}{\delta h_{i j}} \Psi\left(c_{1}, c_{2}, \ldots, c_{D}\right)=D h h^{i j} \frac{\delta}{\delta h} \Psi(h),
$$

where we have used the identity

$$
\delta h=h h^{i j} \delta h_{i j} .
$$

following from the Jacobi formula for determinant of the space-time metric $\delta g=g g^{\mu v} \delta g_{\mu v}$ jointed with the $D+1$ decomposition. Calculating

$$
\frac{\delta h}{\delta c_{n}} \frac{\delta}{\delta h} \Psi(h)=h h^{i j} \frac{\delta h_{i j}}{\delta c_{n}} \frac{\delta}{\delta h} \Psi(h),
$$

and applying this result in (100), one receives

$$
\frac{\delta}{\delta h_{i j}} \Psi\left(c_{1}, c_{2}, \ldots, c_{D}\right)=h h^{i j}\left(\sum_{n=1}^{D} \frac{\delta c_{n}}{\delta h_{i j}} \frac{\delta h_{i j}}{\delta c_{n}}\right) \frac{\delta}{\delta h} \Psi(h),(104)
$$

what after calculation of the sum

$$
\sum_{n=1}^{D} \frac{\delta c_{n}}{\delta h_{i j}} \frac{\delta h_{i j}}{\delta c_{n}}=\sum_{n=1}^{D} \frac{\delta c_{n}}{\delta c_{n}}=\sum_{n=1}^{D} 1=\underbrace{1+\ldots+1}_{D \text { times }}=D,
$$

gives

$$
G_{i j k l} \frac{\delta^{2}}{\delta h_{i j} \delta h_{k l}}=D G_{i j k l} h^{i j} h^{k l} h^{2} \frac{\delta^{2}}{\delta h^{2}} .
$$

Consequently, the reduction leads to

$$
G_{i j k l} h^{i j} h^{k l}=-\frac{D(D-2)}{2} h^{-1 / 2},
$$

where we have used the relations $h^{a b} h_{b c}=\delta_{c}^{a}$ and $\delta_{a}^{a}=D$. Joining (106) and (107), one obtains finally

$$
G_{i j k l} \frac{\delta^{2}}{\delta h_{i j} \delta h_{k l}}=-\frac{D^{2}(D-2)}{2} h^{3 / 2} \frac{\delta^{2}}{\delta h^{2}},
$$

which leads to the one-dimensional objective quantum geometrodynamics

$$
h^{1 / 2}\left[\begin{array}{c}
\kappa D^{2}(D-2) h \frac{\delta^{2}}{\delta h^{2}} \\
-\frac{1}{2 \kappa}\left({ }^{(D)} R-2 \Lambda-2 \kappa \varrho[h]\right)
\end{array}\right] \Psi(h)=0,
$$

or in simplified form

$$
\left[\frac{\delta^{2}}{\delta h^{2}}-\frac{{ }^{(D)} R-2 \Lambda-2 \kappa \varrho[h]}{2 \kappa^{2} D^{2}(D-2)} \frac{1}{h}\right] \Psi(h)=0,
$$

which is correct for $D \neq 0,2$, and whenever $h \neq 0$.

\section{Euclidean Dirac Equation}

Objective quantum geometrodynamics (109) is the Klein-Gordon equation

$$
\left(\frac{\delta^{2}}{\delta h^{2}}+\omega^{2}\right) \Psi=0
$$

where

$$
\omega^{2}=-\frac{{ }^{(D)} R-2 \Lambda-2 \kappa \varrho[h]}{2 \kappa^{2} D^{2}(D-2)} \frac{1}{h}=\frac{K^{2}-K^{i j} K_{i j}}{2 \kappa^{2} D^{2}(D-2)} \frac{1}{h},
$$

which can be positive, negative or vanishing identically. The Euler-Lagrange equation of motion (111) arises from stationarity of the action

$$
S[\Psi]=\int \delta h L\left(\Psi, \frac{\delta \Psi}{\delta h}\right),
$$

where $L$ is the Lagrange function 


$$
L=\frac{1}{2} \Pi_{\Psi}^{2}-\frac{\omega^{2}}{2} \Psi^{2},
$$

and the momentum $\Pi_{\Psi}$ conjugated to the classical scalar field $\Psi$ is

$$
\Pi_{\Psi}=\frac{\partial L}{\partial\left(\frac{\delta \Psi}{\delta h}\right)}=\frac{\delta \Psi}{\delta h}
$$

Since $S[\Psi]$ is a functional of $\Psi, h$ behaves as a parameter in $\omega$ and is unessential in derivation of the equations of motion

$$
\frac{\partial L}{\partial \Psi}-\frac{\delta}{\delta h} \frac{\partial L}{\partial\left(\frac{\delta \Psi}{\delta h}\right)}=0, \int \delta\left(\frac{\partial L}{\partial \Psi} \delta \Psi\right)=\left.\frac{\partial L}{\partial \Psi} \delta \Psi\right|_{0}=0, \text { (116) }
$$

where the standard vanishing boundary condition was taken ad hoc. The first equation in (116) gives (111). Applying $\Pi_{\Psi}$, (111) becomes

$$
\frac{\delta \Pi_{\Psi}}{\delta h}+\omega^{2} \Psi=0
$$

and (115) and (117) are the Hamilton canonical equations of motion

$$
\begin{aligned}
& \frac{\delta}{\delta h} \Psi=\frac{\delta}{\delta \Pi_{\Psi}} H\left(\Psi, \Pi_{\Psi}\right), \\
& \frac{\delta}{\delta h} \Pi_{\Psi}=-\frac{\delta}{\delta \Psi} H\left(\Psi, \Pi_{\Psi}\right),
\end{aligned}
$$

where the Hamilton function $H\left(\Psi, \Pi_{\Psi}\right)$ is obtained by the Legendre transformation

$$
H\left(\Psi, \Pi_{\Psi}\right)=\Pi_{\Psi} \frac{\delta \Psi}{\delta h}-L\left(\Psi, \frac{\delta \Psi}{\delta h}\right)=\frac{1}{2} \Pi_{\Psi}^{2}-\frac{\omega^{2}}{2} \Psi^{2} .
$$

If one introduces the two-component field $\Phi=\left[\begin{array}{ll}\Pi_{\Psi} & \Psi\end{array}\right]$, then the canonical equations of motion (118) became the one-dimensional Dirac equation

$$
\left(-i \sigma_{y} \frac{\delta}{\delta h}-M\right) \Phi=0, M=\left[\begin{array}{cc}
-1 & 0 \\
0 & -\omega^{2}
\end{array}\right],
$$

where $M$ is the mass matrix of the field $\Phi$. The Pauli matrix $\sigma_{y}$ satisfies

$$
\sigma_{y}=\left[\begin{array}{cc}
0 & -i \\
i & 0
\end{array}\right], \sigma_{y}^{2}=\mathrm{I}_{2},\left\{\sigma_{y}, \sigma_{y}\right\}=2 \mathrm{I}_{2}, \mathrm{I}_{2}=\left[\begin{array}{ll}
1 & 0 \\
0 & 1
\end{array}\right],
$$

which is the Clifford algebra $\mathcal{C}_{2,0}(\mathbb{C})$ over the complex vector space $\mathbb{C}^{2}$

$$
\mathcal{C} \ell_{2}(\mathbb{C})=\mathcal{C} \ell_{0}(\mathbb{C}) \otimes \mathrm{M}_{2}(\mathbb{C}) \cong \mathrm{M}_{2}(\mathbb{C})=\mathbb{C} \oplus \mathbb{C},
$$

where $\mathcal{C} \ell_{n} \equiv \mathcal{C} \ell_{n, 0}$, and $\mathrm{M}_{2}(\mathbb{C})$ denotes algebra of all $2 \times 2$ matrices over $\mathbb{C} \cdot \mathcal{C} \ell_{2,0}(\mathbb{C})$ possesses a twodimensional complex representation. Restriction to the pinor group $\operatorname{Pin}_{2,0}(\mathbb{R})$ yields a complex representation of two-dimensional pinor group, that is the two-dimensional spinor representation, whereas restriction to the spinor group $\operatorname{Spin}_{2,0}(\mathbb{R})$ splits $\mathcal{C}_{1,1}(\mathbb{R})$ onto a sum of two half spin representations of dimension 1 , that is the one dimensional Weyl representations. There is the isomorphism

$$
\operatorname{Spin}_{2,0}(\mathbb{R}) \cong U(1) \cong S O(2),
$$

and $\operatorname{Spin}_{2,0}(\mathbb{R})$ acts on a 1-sphere $S^{1}$ in such a way that one has a fibre bundle with fibre $\operatorname{Spin}_{1,0}(\mathbb{R})$

$$
\operatorname{Spin}_{1,0}(\mathbb{R}) \rightarrow \operatorname{Spin}_{2,0}(\mathbb{R}) \rightarrow S^{1},
$$

and the homotopy sequence is

$$
\pi_{1}\left(\operatorname{Spin}_{1,0}(\mathbb{R})\right) \rightarrow \pi_{1}\left(\operatorname{Spin}_{2,0}(\mathbb{R})\right) \rightarrow \pi_{1}\left(S^{1}\right)
$$

Moreover, $\quad \mathcal{C} \ell_{2}(\mathbb{C})$ can be generated by complexification

$$
\mathcal{C} \ell_{2}(\mathbb{C}) \cong \mathcal{C} \ell_{1,1}(\mathbb{R}) \otimes \mathcal{C} \ell_{0}(\mathbb{C}),
$$

where $\mathcal{C} \ell_{1,1}(\mathbb{R})$ is the four-dimensional Clifford algebra over the real vector space $\mathbb{R}^{2,0}$

$$
\mathcal{C} \ell_{1,1}(\mathbb{R}) \cong \mathrm{M}_{2}(\mathbb{R}) \otimes \mathcal{C} \ell_{0}(\mathbb{R}) \cong \mathrm{M}_{2}(\mathbb{R}),
$$

with $\mathrm{M}_{2}(\mathbb{R})$ being algebra of $2 \times 2$ matrices over $\mathbb{R}$, and

$$
\mathcal{C} \ell_{0}(\mathbb{R})=\mathbb{R}, \mathcal{C} \ell_{0}(\mathbb{C})=\mathbb{C} .
$$

$\mathcal{C} \ell_{1,1}(\mathbb{R})$ can be decomposed into a direct sum of central simple algebras isomorphic to matrix algebra over $\mathbb{R}$

$$
\begin{aligned}
& \mathcal{C} \ell_{1,1}(\mathbb{R})=\mathcal{C} \ell_{1,1}^{+}(\mathbb{R}) \oplus \mathcal{C} \ell_{1,1}^{-}(\mathbb{R}), \\
& \mathcal{C} \ell_{1,1}^{ \pm}(\mathbb{R})=\frac{1 \pm \gamma}{2} \mathcal{C} \ell_{1,1}(\mathbb{R}) \cong \mathbb{R},
\end{aligned}
$$

as well as into a tensor product

$$
\begin{aligned}
& \mathcal{C} \ell_{1,1}(\mathbb{R})=\mathcal{C} \ell_{2,0}(\mathbb{R}) \otimes \mathcal{C} \ell_{0,0}(\mathbb{R}), \\
& \mathcal{C} \ell_{2,0}(\mathbb{R})=\mathrm{M}_{2}(\mathbb{R}) \otimes \mathcal{C} \ell_{0,0}(\mathbb{R}) \cong \mathrm{M}_{2}(\mathbb{R}) .
\end{aligned}
$$

\section{Field Quantization}

The Euclidean Dirac equation (120) can be quantized

$$
\left(-i \sigma_{y} \frac{\delta}{\delta h}-M\right) \hat{\Phi}=0,
$$

according to the canonical commutation relations for bosonic fields

$$
\begin{aligned}
& i\left[\hat{\Pi}_{\Psi}\left[h^{\prime}\right], \hat{\Psi}[h]\right]=\delta\left(h^{\prime}-h\right), \\
& i\left[\hat{\Pi}_{\Psi}\left[h^{\prime}\right], \hat{\Pi}_{\Psi}[h]\right]=0, \\
& i\left[\hat{\Psi}\left[h^{\prime}\right], \hat{\Psi}[h]\right]=0,
\end{aligned}
$$

due to the one-dimensionality, that is the lack of difference between bosonic and fermionic statistics which defines the particles called axions. Therefore, the 
quantized Euclidean field theory (131) describes gravitons as axions.

Applying the Fock space of creation and annihilation operators, that is

$$
\hat{\Phi}=Q \Re, Q=\frac{1}{\sqrt{2}}\left[\begin{array}{cc}
\sqrt{1 / \omega} & \sqrt{1 / \omega} \\
-i \sqrt{\omega} & i \sqrt{\omega}
\end{array}\right],
$$

where $\mathcal{B}=\mathscr{B}[h]$ is a dynamical repère

$$
\mathscr{B}=\left\{\begin{array}{c}
{\left[\begin{array}{c}
G[h] \\
G^{\dagger}[h]
\end{array}\right]:\left[G\left[h^{\prime}\right], G^{\dagger}[h]\right]=\delta\left(h^{\prime}-h\right),} \\
{\left[G\left[h^{\prime}\right], G[h]\right]=0}
\end{array}\right\},
$$

one receives the following equations of motion

$$
\frac{\delta \mathfrak{B}}{\delta h}=\mathrm{X} \Re, \quad \mathrm{X}=\left[\begin{array}{cc}
-i \omega & \frac{1}{2 \omega} \frac{\delta \omega}{\delta h} \\
\frac{1}{2 \omega} \frac{\delta \omega}{\delta h} & i \omega
\end{array}\right]
$$

Supposing existence of the repère $\mathfrak{F}$ determined by the Bogoliubov transformation

$$
\mathfrak{F}=\left[\begin{array}{cc}
u & v \\
v^{*} & u^{*}
\end{array}\right] \mathfrak{B},|u|^{2}-|v|^{2}=1,
$$

and the Heisenberg equations of motion

$$
\frac{\delta \mathfrak{F}}{\delta h}=\left[\begin{array}{cc}
-i \Omega & 0 \\
0 & i \Omega
\end{array}\right] \mathfrak{F},
$$

the equations (135) become the vector equation

$$
\frac{\delta \mathrm{b}}{\delta h}=\mathrm{Xb}, \mathrm{b}=\left[\begin{array}{l}
u \\
v
\end{array}\right],
$$

while identically $\Omega \equiv 0$. Therefore, $\mathfrak{F}$ is the static initial data $(I)$ Fock repère

$$
\mathfrak{F}=\left\{\left[\begin{array}{l}
\mathrm{G}_{I} \\
\mathrm{G}_{I}^{\dagger}
\end{array}\right]:\left[\mathrm{G}_{I}, \mathrm{G}_{I}^{\dagger}\right]=1,\left[\mathrm{G}_{I}, \mathrm{G}_{I}\right]=0\right\},
$$

wherein the vacuum state $|0\rangle$ is automatically stable

$$
G_{I}|0\rangle=0,\langle 0| G_{I}^{\dagger}=0
$$

Integrability of the equations (138) is crucial. The Bogoliubov transformation (136) suggests using of the superfluid parametrization

$$
u=e^{i \theta} \cosh \phi, \quad v=e^{i \theta} \sinh \phi,
$$

with the angles

$$
\theta= \pm i \int_{h_{I}}^{h} \omega^{\prime} \delta h^{\prime}, \quad \phi=\ln \sqrt{\left|\frac{\omega_{I}}{\omega}\right|},
$$

where $\omega^{\prime}=\omega\left(h^{\prime}\right)$ and $\omega_{I}$ is the initial datum of $\omega$

$$
\omega_{I}=-\frac{1}{\kappa \sqrt{2} D \sqrt{D-2}},
$$

which yield the Bogoliubov coefficients $u=\frac{\mu+1}{2 \sqrt{\mu}} \exp \left\{i \int_{h_{I}}^{h} \omega^{\prime} \delta h^{\prime}\right\}, v=\frac{\mu-1}{2 \sqrt{\mu}} \exp \left\{-i \int_{h_{I}}^{h} \omega^{\prime} \delta h^{\prime}\right\}$

where $\mu=\frac{\omega}{\omega_{I}}$. Consequently, the integrability problem is solved

$$
\hat{\Phi}=\mathrm{QG} F
$$

where $\mathrm{G}$ is the monodromy matrix

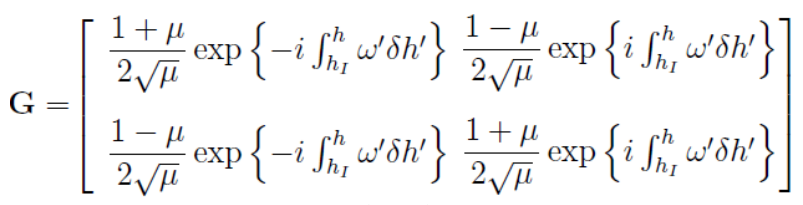

The initial data condition $\omega=\omega_{I}$ generates the equivalent equations for the initial manifold

$$
{ }^{(3)} R^{(I)}-2 \Lambda-2 \kappa \varrho_{I}=h_{I}, K_{i j}^{(I)} K^{(I) i j}-K^{(I) 2}=h_{I} \text {. }
$$

In such a situation, the quantum evolution (111) becomes

$$
\left(\frac{\delta^{2}}{\delta h_{I}^{2}}-\frac{1}{2 \kappa^{2} D^{2}(D-2)}\right) \Psi\left(h_{I}\right)=0,
$$

and with the suitable boundary conditions

$$
\Psi\left(h_{I}=h_{0}\right)=\Psi_{0},\left.\frac{\delta \Psi\left(h_{I}\right)}{\delta h_{I}}\right|_{h_{I}=h_{0}}=\Pi_{\Psi}^{0},
$$

can be solved straightforwardly

$$
\begin{aligned}
\Psi\left(h_{I}\right)= & \Psi_{0} \cosh \left\{\frac{h_{I}-h_{0}}{\kappa \sqrt{2} D \sqrt{D-2}}\right\} \\
& +\kappa \sqrt{6} \Pi_{\Psi}^{0} \sinh \left\{\frac{h_{I}-h_{0}}{\kappa \sqrt{2} D \sqrt{D-2}}\right\} .
\end{aligned}
$$

\section{Relation to String Theory}

Objective quantum gravity can be efficiently applied to the estimation of the spatial dimension $D$. Writing the evolution equation (110) as the algebraic equation for $D$

$$
\begin{aligned}
& D^{2}(D-2) \\
= & \frac{1}{2 \kappa^{2}}\left({ }^{(D)} R-2 \Lambda-2 \kappa \varrho\right)\left(\frac{h}{\Psi} \frac{\delta^{2} \Psi}{\delta h^{2}}\right)^{-1} \equiv \Delta,
\end{aligned}
$$

for the case of $\Delta>0$, its real solution is

$$
\begin{aligned}
D= & \frac{2}{3}+\frac{1}{3}\left(8+\frac{27}{2} \Delta-\frac{3}{2} \sqrt{3} \sqrt{32 \Delta+27 \Delta^{2}}\right)^{1 / 3} \\
& +\frac{1}{3}\left(8+\frac{27}{2} \Delta+\frac{3}{2} \sqrt{3} \sqrt{32 \Delta+27 \Delta^{2}}\right)^{1 / 3},
\end{aligned}
$$

and, consequently, the physical values of the space dimension are

$$
D>2 \rightarrow D=3,4,5 \text {, etc, }
$$


what gives the following lower bound

$$
\Delta \geq 9 \text {. }
$$

In the manifestly non-physical case $\Delta<0$, one receives

$$
\begin{aligned}
D= & \frac{2}{3}+\frac{1}{3}\left(8-\frac{27}{2}|\Delta|-\frac{3}{2} \sqrt{3} \sqrt{-32|\Delta|+27 \Delta^{2}}\right)^{1 / 3} \\
& +\frac{1}{3}\left(8-\frac{27}{2}|\Delta|+\frac{3}{2} \sqrt{3} \sqrt{-32|\Delta|+27 \Delta^{2}}\right)^{1 / 3},
\end{aligned}
$$

and, consequently, the physical value of the spatial dimension is

$$
D \in(-\infty, 2) /\{0\} \rightarrow D=1 .
$$

However, this gives $\Delta=-1$, what is a physically irrelevant result.

In the case $\Delta=0$, the possibilities for the spatial dimension are

$$
D=0,2
$$

and $D=2$ is the physically relevant value. However, this case is inconsistent with the model of quantum gravity.

In the light of the established lower bound (154), one can suggest that $\Delta=D^{2}(D-2)$ is a dimension of a certain effective space, and $\Delta+1$ is the dimension of the effective space-time. By the spirit of string theory, one can suggest that such an effective space-time is the space-time wherein strings and superstrings live. Because in the case of strings the upper bound for the space-time dimension is 26, the dimension of the effective space is at most $\Delta_{\text {strings }}=25$. When one applies supersymmetry, then the upper bound for space-time dimensionality is 11 , that is $\Delta_{\text {super strings }}=10$. Therefore, the minimal value of the spatial dimension $D$ must be equal to 3 , and the cases of 0-, 1- and 2- dimensional classical spaces are non-physical. For this reason, the emergent string theory in the effective space-time is the relation between the objective quantum gravity and string theory.

The minimal dimension of the effective space-time, following from the lower bound (154), would be 10 . Interestingly, for $\Delta \leq 25$ the upper bound for the space dimension is

$$
\Delta_{\text {strings }} \underset{\approx}{<3.7643,}
$$

which in the light of the lower bound (153) and the fact that the spatial dimension $D$ should be a natural number leads to the conclusion that the value 4 is the stable dimension of the classical space-time. Similarly, when $\Delta \leq 11$ one has the following upper bound

$$
\Delta_{\text {super strings }} \underset{\approx}{<3.0647 .}
$$

The both received upper bounds (158) and (159) can be interpreted as the syndrome of the existence of comparatively small values of the fluctuation to the stable spatial dimension, which is 3 .

Apparently, this is fully consistent with the method of dimensional regularization, which was applied by the obel Laureates in Physics Gerardus't Hooft and Martinus Justinus Godefridus Veltman [31] to elucidate the problem of renormalization of gauge field theories through the efficient regularization of the divergent loop integrals in the evaluation of the Feynman diagrams. In this regularization procedure, the dimension of space-time is $4+\epsilon$, where $\epsilon$ is the fluctuation to the spatial dimension which goes to the limit value $0^{-}$. Consequently, the formula $D=3+\epsilon$ gives

$$
\begin{aligned}
& \epsilon_{\text {strings }} \underset{\approx}{<0.7643,} \\
& \epsilon_{\text {super strings }} \underset{\approx}{<0.0647,}
\end{aligned}
$$

and existence of supersymmetry significantly restricts these fluctuations

$$
\frac{\epsilon_{\text {super strings }}}{\epsilon_{\text {strings }}} \approx 0.0846 \text {. }
$$

If one applies the method of dimensional regularization, then one can put straightforwardly $D=3+\epsilon$ in the equation (152), and the fluctuation of spatial dimension is given by the dimension of the effective space

$$
\begin{aligned}
\epsilon= & -\frac{7}{3}+\frac{1}{3}\left(8+\frac{27}{2} \Delta-\frac{3}{2} \sqrt{3} \sqrt{32 \Delta+27 \Delta^{2}}\right)^{1 / 3} \\
& +\frac{1}{3}\left(8+\frac{27}{2} \Delta+\frac{3}{2} \sqrt{3} \sqrt{32 \Delta+27 \Delta^{2}}\right)^{1 / 3},
\end{aligned}
$$

and, moreover,

$$
\Delta=(3+\epsilon)^{3}-2(3+\epsilon)^{2}=\epsilon^{3}+7 \epsilon^{2}+15 \epsilon+9 .
$$

Therefore, the change in the dimension of the classical space by the value $\epsilon=1$ corresponds to the change $\Delta=32$ in the dimension of the effective space. This means that even a comparatively small change in the spatial dimension results in a large change in the effective space dimension. The classical space-time of dimension 5 corresponds to the effective space-time of dimension 33, and when the dimension of the effective space-time is limited to $\Delta \gg 32$, then the spatial dimension fluctuation is $\epsilon \gg 1$.

When one considers the Yang-Mills theory with the number of colors $N=\sqrt{D+1}=\sqrt{4+\epsilon}$, then making Taylor power series expansion for $\epsilon \ll 1$ one has $N=2+\frac{\epsilon}{4}+O\left(\epsilon^{2}\right)$, and the gauge group is $G=S U\left(2+\frac{\epsilon}{4}\right) . \quad$ The relation $\Delta=N^{2}\left(N^{4}+7\right)-\left(5 N^{4}+3\right)$ in the large-N limit becomes $\Delta \approx N^{6}-5 N^{4}$, whereas for small $N$ one has $\Delta=7 N^{2}-3$. Applying $N=\sqrt{D+1}$ in (152), one receives the formula

$$
N=\left[\begin{array}{l}
\frac{5}{3}+\frac{1}{3}\left(8+\frac{27}{2} \Delta-\frac{3}{2} \sqrt{3} \sqrt{32 \Delta+27 \Delta^{2}}\right)^{1 / 3} \\
+\frac{1}{3}\left(8+\frac{27}{2} \Delta+\frac{3}{2} \sqrt{3} \sqrt{32 \Delta+27 \Delta^{2}}\right)^{1 / 3}
\end{array}\right]^{1 / 2},
$$

which for a small $\Delta$ behaves as 


$$
N_{\Delta \ll 1}=\sqrt{3}+\frac{\Delta}{8 \sqrt{3}}+O\left(\Delta^{3}\right),
$$

whereas for a large $\Delta$ is

$$
N_{\Delta \gg 1}=\Delta^{1 / 6}+O\left(\frac{1}{\Delta^{1 / 6}}\right) .
$$

Taking into account (158) and (159), one sees that for the case of strings the number of colors is bounded by $N_{\text {strings }}<2.1827$, whereas for the case of superstings the bound is slightly different $N_{\text {super strings }}<2$.0161. In this manner, the hypothetical gauge theory is fully consistent with the scenario of the effective space-time if and only if $N=1,2$, that is the spatial dimension is 0 or 3 and, therefore, the full physical consistency is established for $N=2$ and $D=3$, for which the dimension of the effective space is $\Delta=9$. Then, the gauge group is $G=S U(2)$, that is the corresponding Yang-Mills theory describes the weak interactions of the Standard Model.

\section{Discussion}

In the presented scenario, objective quantum geometrodynamics allows to formulate quantum gravity as a quantum field theory and, moreover, make the relationships with gauge theories and string theory throughout the effective space-time. It is clear that the presented construction in itself is non-trivial, because allows to find the straightforward relationships between an abstractive formalism of quantum gravity and high energy physics. Moreover, the theory is mathematically extraordinary simple, because given by the Euclidean Dirac equation, whereas the gravitons are defined as axions. In this manner, the presented model to quantum gravity possesses a manifestly phenomenological meaning. We hope for further developments of this approach. The updates and developments to this model were recently published in the author's monograph [32].

\section{References}

[1] Giulini, D. \& Kiefer, C. (2007). The Canonical Ap- proach to Quantum Gravity: General Ideas and Geometrodynamics, In: Approaches to Fundamental Physics: An Assessment of Current Theoretical Ideas, I.-O. Stamatescu and E. Seiler, (Eds.), Lect. Notes Phys. 721, pp. 131-150, Springer, New York.

[2] Kiefer, C. (2009). Quantum geometrodynamics: whence, whither?. Gen. Rel. Grav. 41, (2009) 877-901.

[3] 't Hooft, G. \& Veltman, M.J.G. (1973). Dia- grammar, CERN, Geneva.

[4] Veltman, M.J.G. (1976). Quantum Theory of Gravitation, In: Methods in Field Theory. Proc. Les Houches, Session XXVIII, R. Balian and J. Zinn-Justin, (Eds.), pp. 265-328, North Holland, Ams- terdam.

[5] Bern, Z.; Carrasco, J.J.M. \& Johansson, H. (2010). Per- turbative Quantum Gravity as a Double Copy of Gauge Theory. Phys. Rev. Lett. 105, (2010) 061602.

[6] Wadia, S.R. (2008). String Theory: A Framework for Quan- tum Gravity and Various Applications. E-print: arXiv:0809.1036 [grqc].

[7] Blau, M. \& Theisen, S. (2009). String theory as a theory of quantum gravity: a status report. Gen. Rel. Grav. 41, (2009) 743755.
[8] Giddings, S.B. (2011). Is string theory a theory of quantum gravity?. E-print: arXiv:1105.6359 [hep-th].

[9] Ashtekar, A. (2007). An Introduction to Loop Quantum Gravity Through Cosmology. Nuovo Cim. B 122, (2007) 135-155.

[10] Perez, A. (2009). Loop quantum gravity: An introduction. AIP Conf. Proc. 1132, (2009) 386-428.

[11] Domagala, M; Giesel, K.; Kaminski, W. \& Lewandowski, J. (2010). Gravity quantized: Loop Quantum Gravity with a Scalar Field. Phys. Rev. D 82, (2010) 104038.

[12] Rovelli, C. (2011). Loop quantum gravity: the first twenty five years. Class. Quant. Grav. 28, (2011) 153002.

- (2011). A new look at loop quantum gravity. Class. Quant. Grav. 28, (2011) 114005.

[13] Oriti, D. (Ed.) (2009). Approaches To Quantum Gravity. To- ward A New Understanding Of Space, Time And Matter, Cambridge University Press, Cambridge.

[14] Wheeler, J.A. (1957). On the Nature of Quantum Geometrodynamics. Ann. Phys. 2, (1957) 604-614.

- (1962). Geometrodynamics, Academic Press, New York.

- (1964). Geometrodynamics and the Issue of the Final State, In: Relativity, Groups, and Topology. Lectures Delivered at Les Houches During the 1963 Session of the Summer School of Theoretical Physics, University of Grenoble, C. DeWitt and B. DeWitt, (Eds.), pp. 317-501, Gordon and Breach, New York.

- (1968). Superspace and the Nature of Quantum Geometrodynam- ics, In: Battelle Rencontres. 1967 Lectures in Mathematics and Physics,

C.M. DeWitt and J.A. Wheeler, (Eds.), pp. 242-308, W.A. Benjamin, New York.

- (1968). Einsteins Vision, Springer-Verlag, New York.

- (1970). Superspace, In: Analytic Methods in Mathematical Physics,

R.P. Gilbert and R. Newton, (Eds.), pp. 335-378, Gordon and Breach, New York.

[15] DeWitt, B.S. (1967). Quantum Theory of Gravity I. The Canonical Theory. Phys. Rev. 160, (1967) 1113-1148.

- (1967). Quantum Theory of Gravity II. The Manifestly Covariant Theory. Phys. Rev. 160, (1967) 1195-1239.

- (1967). Quantum Theory of Gravity III. Applications of the Co- variant Theory. Phys. Rev. 160, (1967) 1239-1256.

[16] Dirac, P.A.M. (1964). Lectures on Quantum Mechanics, Belfer Graduate School of Science, Yeshiva University.

- (1959). Fixation of Coordinates in the Hamiltonian Theory of Grav- itation, Phys. Rev. 114, (1959) 924-930.

- (1959). Energy of the Gravitational Field. Phys. Rev. Lett. 2, (1959) 368-371.

- (1958). Generalized Hamiltonian Dynamics. Proc. R. Soc. A 246 , (1958) 326-332

- (1958). The Theory of Gravitation in Hamiltonian Form. Proc. R. Soc. A 246, (1958) 333-343.

- (1950). Generalized Hamiltonian Dynamics. Can. J. Math. 2, (1950) 129-148.

- (1949). Forms of Relativistic Dynamics. Rev. Mod. Phys 21, (1949) 392-399.

[17] Arnowitt, R.; Deser, S. \& Misner, C.W. (1961). The Dynamics of General Relativity, In: Gravitation: an introduction to current research, L. Witten, (Ed.), pp. 227-265, John Wiley \& Sons, New York.

[18] Hartle, J.B. \& Hawking, S.W. (1983). Wave func- tion of the Universe. Phys. Rev. D 28, (1983) 2960-2975.

[19] Halliwell, J.J. \& Hawking, S.W. (1985). Origin of structure of the Universe. Phys. Rev. D 31, (1985) 1777-1791.

[20] Coleman, S.; Hartle, J.B.; Piran, T. \& Weinberg, S. (Eds.) (1991), Quantum Cosmology and Baby Universes, World Scien- tific, Singapore.

[21] Hartle, J.B.; Hawking, S.W. \& Hertog T. (2008). Clas- sical universes of the no-boundary quantum state. Phys. Rev. D 77, (2008) 123537.

- (2008). No-Boundary Measure of the Universe. Phys. Rev. Lett. 100, (2008) 201301.

[22] Glinka, L.A. (2007). Quantum Information from Graviton-Matter Gas. SIGMA 3, (2007) 087, 13 pages.

- (2007). Preliminaries in Many-Particle Quantum Gravity. Einstein- Friedmann Spacetime. E-print: arXiv:0711.1380 [gr-qc] - (2008). Multiparticle Quantum Cosmology, In: Frontiers of Fun- damental and Computational Physics. 9th International Symposium, Udine and Trieste, Italy 7-9 January 2008, B.G. Sidharth, F. Honsell, O. Mansutti, K. Sreenivasan, and A. De 
Angelis (Eds.), AIP Conf. Proc. 1018, (2008) 94-99, American Institute of Physics, New York.

- (2008). Quantum gravity as the way from spacetime to space quan- tum states thermodynamics. New Adv. Phys. 2, (2008) 1-62 - (2008). 1D Global Bosonization of Quantum Gravity. E-print: arXiv:0804.3516 [gr-qc].

- (2008). Many-Particle Quantum Cosmology, In: Supersymmetries and Quantum Symmetries (SQS'07): Proceedings of International Workshop, held in Dubna, Russia, July 30 - August 4, 2007, E. Ivanov and S. Fedoruk (Eds.), pp. 406-411, JINR, Dubna. - (2009). Novel solution of Wheeler-DeWitt theory. E-print: arXiv:0906.3825 [gr-qc].

- (2009). Thermodynamics of space quanta models quantum gravity. E-print: arXiv:0906.3827 [gr-qc].

- (2009). Macrostates thermodynamics and its stable classical limit in Global One-Dimensional Quantum General Relativity. Concepts Phys. 6, (2009) 19-42.

- (2009). New Approach to Quantization of Cosmological Models. Grav. Cosmol. 15, (2009) 317-322

- (2010). Global One-Dimensionality conjecture within Quantum General Relativity. Grav. Cosmol. 16, (2010) 7-15.

- (2011). On Quantum Cosmology as Field Theory of Bosonic String Mass Groundstate. Oriental J. Physics 3(1), pp. 1-10.

- (2014). On the Residual Effective Potential within Global One- Dimensional Quantum Gravity, J. Astroph. Aeros. Technol. 2(1), 1000103.
[23] Weinberg, S. (1972). Gravitation and Cosmology. Princi- ples and Applications of the General Theory of Relativity, John Wiley \& Sons, New York.

[24] Misner, C.W.; Thorne, K.S. \& Wheeler, J.A. (1973). Gravitation, W.H. Freeman, San Francisco.

[25] Landau, L.D. \& Lifshitz, E.M. (1994). Course of Theoretical Physics, Vol 2. The Classical Theory of Fields, 4th English ed., Butterworth Heinemann, Amsterdam.

[26] Carroll, S. (2004). Space-time and Geometry. An Introduction to General Relativity, Addison-Wesley, San Francisco.

[27] Poisson, E. (2004). A Relativist's Toolkit. The Mathematics of Black-Hole Mechanics, Cambridge University Press, Cambridge.

[28] DeWitt, B. (2003). The Global Approach to Quantum Field Theory, Vols. 1-2, Int. Ser. Monogr. Phys. 114, Clarendon Press, Ox- ford.

[29] Masahiro, S. (1987). Nash Manifolds, Lect. Notes Math. 1269, Springer, New York.

[30] Giulini, D. (2009). The Superspace of geometrodynamics. Gen. Rel. Grav. 41, (2009) 785-815.

[31] 't Hooft, G. \& Veltman, M. (1972). Regulariza- tion and renormalization of gauge fields. Nuclear Physics B 44, (1972) 189-213.

[32] Glinka, L.A. (2012). Æthereal Multiverse: A New Unifying Theoretical Approach to Cosmology, Particle Physics, and Quantum Gravity, Cambridge International Science Publishing, Great Abington. 